\title{
La confiance
}

\section{Anna Sax}

Lic. oec. publ., MHA, membre de la rédaction

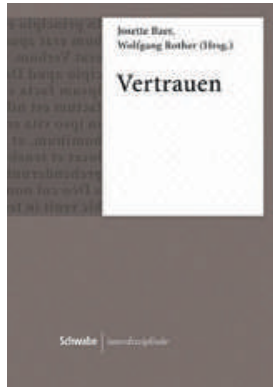

Josette Baer, Wolfgang Rother Vertrauen

Basel: Schwabe Verlag; 2015. ISBN 978-3-7965-3451-5 231 Seiten. 48 CHF.

\footnotetext{
* Les genres masculin et féminin ne sont pas choisis par hasard, bien que de nombreuses déclarations pourraient également s'appliquer dans une relation médecin-patient où ceux-ci sont inversés.
}

anna.sax[at]saez.ch
D’après le sondage représentatif effectué chaque année par Reader's Digest, les médecins jouissent d'une grande confiance dans la population. En 2015, 87\% des personnes interrogées en Suisse déclaraient avoir une grande ou une assez grande confiance en le corps médical. Seuls les sapeurs-pompiers (92\%), les pilotes de ligne (91\%), le personnel infirmier (90\%) et les pharmaciens (88\%) jouissent d'une plus grande confiance. Qu'est-ce qui vaut aux médecins - les hommes plus que les femmes - un tel privilège? Pourquoi la population leur fait-elle davantage confiance qu'aux politiciens, journalistes ou conseillers financiers, pour ne prendre que ces exemples?

Historienne de la médecine, Iris Ritzmann propose une réponse dans l'article publié dans l'ouvrage collectif Vertrauen ("La confiance»), paru il y a quelques mois aux éditions Schwabe. L'ouvrage se base sur une série de cours interdisciplinaires donnés en 2014 à l'Université de Zurich. Des spécialistes de divers domaines s'y interrogent sur ce qui détermine la confiance. La question de la confiance en Dieu y est traitée tout comme celle de savoir si l'on peut se fier à un ordinateur ou si la confiance est connue des animaux. On y lit que la confiance est une condition essentielle au fonctionnement de l'économie, mais aussi à la vie des collectivités humaines en général. Créer la confiance peut aussi être l'élément d'une stratégie dont le but est de s'assurer une position de pouvoir. Ce qui nous ramène à l'article d'Iris Ritzmann, que je tiens tout particulièrement à porter à l'attention du public cible de cette revue.

La relation médecin-patient, nous dit Ritzmann, repose depuis toujours sur un déséquilibre des rapports de pouvoir entre le médecin, qui possède les connaissances, et la patiente qui sollicite son aide. ${ }^{*}$ La patiente cède au médecin une responsabilité que celui-ci assume. Selon la thèse de Ritzmann, «La demande de confiance s'inscrit dans une stratégie du médecin visant à fidéliser ses patients.» On attend de la patiente qu'elle fasse confiance au médecin, mais ce n'est pas forcément réciproque. Le médecin n'est pas toujours censé faire confiance à sa patiente, car elle ignore souvent ce qui est bon pour elle. Ritzmann conclut: «Cette notion de confiance décrit une exigence unilatérale du médecin vis-à-vis de son patient et non une relation de partenariat.» Le but du médecin est bien souvent d'«assurer sa position et non d'appliquer une forme de prise en charge dont il a confiance qu'elle guérira le malade». D'un autre côté, il est vrai aussi que beaucoup semblent tout à fait disposés à abdiquer à d'autres, aux experts justement, la tâche de réfléchir. Ils préfèrent faire une confiance aveugle au médecin plutôt que de se responsabiliser.

Iris Ritzmann consacre une partie de son article à la relation du médecin avec ses patientes. Dès le XVIII siècle, plusieurs auteurs ont entrepris de conseiller les médecins sur la manière de gérer les relations avec leur patientèle féminine. Pour gagner la confiance de ces dames, il s'agissait moins de faire valoir sa compétence professionnelle que de se comporter correctement, faire preuve d'assurance et témoigner de la compréhension pour leurs états d'âme. Plus de 200 ans ont passé et pourtant, les lectrices seront surprises en consultant, sur les conseils de Ritzmann, le site web allemand www.ichbinarzt.de. On y trouve - outre des conseils pour s'habiller à la mode -10 suggestions pour

\section{On attend de la patiente qu'elle fasse} confiance au médecin, mais ce n'est pas forcément réciproque.

se donner une "prestance et une assurance» censées créer la confiance. Il n'y est pas question de connaissances médicales, mais de tenue vestimentaire, d'hygiène corporelle, de poignée de mains ferme, de rayonnement positif et d'intérêt témoigné à son vis-à-vis. La photo qui montre une main d'homme en blouse blanche serrant une main féminine qui se dresse vers lui est très révélatrice. Sa légende: «Une poignée de mains franche donne un sentiment de confiance et de sécurité.»

Cela concorde avec l'étude médicale de l'Institut gfs publiée en 2012: pour la satisfaction des patients vis-à-vis de leur médecin, la confiance qu'il leur inspire et l'empathie qu'il leur témoigne sont plus importantes que l'éventuel bénéfice pour leur santé de la consultation. 